\title{
Zur Frage der Hofkunst im Reich und in Frankreich im 14. Jahrhundert
}

\author{
Christian Freigang
}

Über Hofkunst im I4. Jahrhundert zu reflektieren, beruht zunächst auf der axiomatischen Annahme, dass künstlerische Artefakte Kommunikationsmedien darstellen, die soziale Distinktion und Identität vermitteln und insofern dem, Fürstenhof' einen öffentlich wahrnehmbaren Sonderstatus zuweisen. Wie das eigentlich geschieht, darüber ist man sich mangels dokumentarischer Evidenz allerdings nicht ganz im Klaren: Und so lassen sich, wie zuletzt durch Brigitte KurmannSchwarz, Wolfgang Brückle und Malcolm Vale ausgeführt, recht unterschiedliche Definitionen des Begriffs, Hofkunst' finden. ${ }^{1}$ Während er seit den Sechzigerjahren des 20. Jahrhunderts als Epochenbegriff der durch ein ebenso expressives wie elegantes Idiom ausgezeichneten Kunst unter Ludwig IX. von Frankreich galt, ${ }^{2}$ wurde der Terminus von Robert Suckale in einem allgemeinen stilgeschichtlichen Sinne auch auf eine künstlerische Produktion bezogen, die sich auf die Selbstdarstellung verschiedener adelig-höfischer Gruppen bezieht. Dabei erhält das Stilidiom dynastisch-distinktiven Charakter, kennzeichnet programmatisch politische Intentionen verschiedener Parteiungen. ${ }^{3}$ Daneben gibt es die prominent von Martin Warnke vertretene Auffassung vom Hofkünstler, der - institutionell an die Adelshöfe gebunden - sich von den zünftisch verfassten kommunalen Kunstproduktionen bzw. den Ateliers und Werkstätten geistlicher Instanzen

1 Brigitte Kurmann, Der Schrein der hl. Gertrud und das Problem der französischen Hofkunst im 13. Jahrhundert. Stand der Forschung und Probleme, in: Schatz aus den Trümmern. Der Silberschrein von Nivelles und die europäische Hochgotik. Ausst.-Kat. Paris und Köln 1995/96, hg. von Hiltrud Westermann-Angerhausen, Köln 1996, S. 237-249; Wolfgang BRÜCKLE, Revision der Hofkunst. Zur Frage historistischer Phänomene in der ausgehenden Kapetingerzeit und zum Problem des höfischen Stils, in: ZK 63 (2000), S. 404-434; Malcolm VALE, The Princely Court. Medieval Courts and Culture in NorthWestern Europe, 1270-1380, Oxford 2001.

2 Robert Branner, St. Louis and the Court Style in Gothic Architecture, London 1965.

3 Robert Suckale, Die Hofkunst Kaiser Ludwigs des Bayern, München 1993. Bernd CARQuÉ, Stil und Erinnerung. Französische Hofkunst im Jahrhundert Karls V. und im Zeitalter ihrer Deutung (Veröffentlichungen des Max-Planck-Instituts für Geschichte 192), Göttingen 2004. 
abhebt. ${ }^{4}$ Der Hofkünstler verfüge in seiner engen Bindung an den Hof bzw. den Herrscher über besondere Freiheiten und Privilegien, sein Publikum sei insofern exklusiv, als an den Höfen eine durch Bildung, verfeinerte Verhaltenskodizes und Divertissement geprägte „höfische Kultur“ eine besondere Wertschätzung von Raffinement und Virtuosität geherrscht habe.

Wie derartige Klassifizierungen in Bezug auf Friedrich den Schönen zu konkretisieren sind, bleibt indessen äußerst schwierig, denn zu gering sind sein politisches Gewicht und seine Stiftertätigkeit, als dass man den Versuch unternehmen könnte, etwa eine ,Hofkunst unter Friedrich dem Schönen' auch nur umreißen zu wollen. Sicherlich hat er die bemerkenswert anspruchsvolle Wiener Augustinereremitenkirche nahe der Hofburg gestiftet. ${ }^{5}$ Im Licht neuerer Forschung sind die bislang für die Ausführung reklamierten bayerischen Werkmeister - deren Bestellung auf eine gezielte Programmatik des Baus hindeuten könnte - allerdings neuzeitliche Quellenkontaminationen. Auch die Grundrissform des Langhauses wird nunmehr mit guten Gründen mit regionalen Traditionen und nicht mit der Übernahme deutscher Vorbilder in Verbindung gebracht. Vor allem ist der bemerkenswerte Chor mit dem sich erweiternden Polygonschluss das Resultat einer durchgreifenden Planänderung der zweiten Hälfte des i4. Jahrhunderts - also lange nach Friedrichs Tod -, die sich dann sehr deutlich an der Chorerweiterung der Aachener Pfalzkapelle orientiert. Geradezu konträr ist ein weiteres Werk aus der Entourage Friedrichs zu beurteilen, die zu Ehren des hl. Ludwig von Toulouse als Grabstätte seiner Ehefrau Elisabeth von Aragon gestiftete Ludwigskapelle an der Wiener Minoritenkirche. ${ }^{6}$ Hier hat man, wohl zu Recht, vor allem auf süddeutsche Einflüsse verwiesen, für das Portaltympanon vor allem auf eine Skulpturenwerkstatt aus Regensburg. Insbesondere aber die um 1330 entstandene Architektur mit ihren innovativen Maßwerkcouronnements und den angeschärften Birnstabprofilen fungiert als Relais zwischen den architektonischen Verfeinerungen, wie man sie zeitgleich in Südwestdeutschland findet, und der Wiener Architektur des I4. Jahrhunderts. Man könnte von hier aus versuchen, den um 1304 begonnenen Ausbau des Hallenchores des Stephansdoms in seiner zweiten, innovativen Bauphase inklusive seiner hervorragenden Bauskulptur der Zeit um 1320/1340

4 Martin WARNKe, Hofkünstler. Zur Vorgeschichte des modernen Künstlers, Köln 1985.

5 Günther Buchinger - Doris SCHÖN, „... jene, die ihre hände hilfreich zum bau erheben ...: Zur zeitlichen Konkordanz von Weihe und Bauvollendung am Beispiel der Wiener Augustinerkirche und Georgskapelle, in: RIHA Journal 0020 (18. April 2011), URL: www. riha-journal.org/articles/2011/2011-apr-jun/buchinger-schoen-wiener-augustinerkirche (zuletzt aufgerufen am 17.4.2015).

6 Maria PARUCki, Die Wiener Minoritenkirche, Wien - Köln - Weimar 1995. 
dem Engagement Friedrichs zuschreiben und daraus eine spezifische Hofkunst ableiten. Allerdings stünde man vor der Schwierigkeit, forcierte Datierungen in die Lebenszeit Friedrichs vorzunehmen und die bezeugt intensive kommunale Stiftungstätigkeit - der Rat der Stadt hatte auch das Bauamt inne - gegen eine nicht belegte Bautätigkeit Friedrichs auszuspielen.

Das zugegeben Diffizile eines solchen Unterfangens hätte aber insofern seinen Reiz, als für die Person des mächtigen und siegreichen Konkurrenten Friedrichs, Kaiser Ludwig der Bayer, durch Robert Suckale sehr nachdrücklich eine spezifische ,Hofkunst' reklamiert worden ist. ${ }^{7}$ Hier wird sehr dezidiert eingefordert, künstlerischen und insbesondere bildhauerischen ,Stil' als politische Programmatik des Kaisers darzustellen, die instrumentell eingesetzt und entsprechend von oberster Instanz dirigiert worden sei. Ausgehend von einer als exemplarisch für den Hofstil eingestuften Madonnenstatue aus Weiler unternimmt Suckale den Versuch, genuin kunsthistorische Kategorien zu aktualisieren, indem sie aus sich heraus, ohne weitere nachweisbare Kommentierung als herrscherliche Selbstdarstellung zu verstehen seien. Implizit bedeutet das, dass sich jedes politische Handeln im Mittelalter zumindest seit einer bestimmten Zeit auf je unterschiedliche, ja dezidiert distinkte Strategien von ,Hofkunst' stützen müsse. Die politische Programmatik eines solchen Konzeptes von Hofkunst besteht insbesondere darin, den Monumenten und Bildwerken über die Evokation von regionaltypischen Stilformen Bündnisse und Allianzen dauerhaft einzuprägen, damit Affirmation und Widerstand zu kennzeichnen. Einige Beispiele: Der stilistische und motivische Rekurs auf Giottos Navicellamosaik der Berufung Petri in St.-Pierre-le-Jeune in Straßburg beziche sich auf die antiludovizische, prorömische Haltung der Stadt - nicht aber etwa nur auf die Intention des patrizischen Stifters. In Frankfurt am Main, politisch wichtig als Stadt der Königswahl, sei die Madonna am Nordquerhaus der Stifts- und Pfarrkirche St. Bartholomäus, also dem Wahlort der Herrscher, aufgrund ihrer Nähe zur wohl von Ludwig dem Bayern gestifteten Madonna in der Zisterzienserkirche Fürstenfeld als öffentlich gemachtes Bekenntnis der Stadt zum Wittelsbacherherrscher, selbst nach dessen Tod, zu interpretieren. Da die Stadt aber innerhalb der Legitimationskämpfe des Nachfolgers, Kaiser Karl IV., rasch auf dessen Seite umgeschwenkt sei, habe man auch den skulpturalen Stil des wenig später entstandenen Südquerhausportals auf die neue karolinische Linie umstellen müssen - so hat man im Gefolge Suckales die These weiter ausgebaut. ${ }^{8}$ Insgesamt sei die fraglos zu beobachtende Neuausrichtung künstlerischer und architektonischer

7 SuCKale, Hofkunst (wie Anm. 3), S. 81.

8 Jiří FAJT - Markus Hörsch, Zwischen Prag und Luxemburg - Eine Landbrücke in den Westen, in: Karl IV. Kaiser von Gottes Gnaden. Kunst und Repräsentation des Hauses 
Tendenzen unter Karl IV. aus einer stilistischen Opposition gegen seinen Erzfeind Ludwig entstanden. ${ }^{9}$ In Verbindung mit Suckales ebenfalls epochalem Werk zur französischen gotischen Architektur des I2. und 13. Jahrhunderts ${ }^{10}$ wurde hier eine umfassende Geschichte der spätmittelalterlichen Kunststile als politisches Instrument vorgelegt. Diese reizvolle Synthese fand ihre aktuelle Fortführung, denn die große Ausstellung zu Karl IV. in Prag und New York 2006 mit dem programmatischen Titel ,Kunst und Repräsentation' schlug den Bogen bis Kaiser Sigismund. So entstehe aus der antiludovizischen Neuausrichtung der Künste unter Karl IV. ein karolinischer Reichsstil, der sich als Universalidiom im sogenannten Internationalen Stil um 1400 manifestiere. " Trotz aller stupenden Kennerschaft und vor allem der emanzipatorisch grundierten Zielsetzung, Kunst als politisches Instrument analysierbar zu machen, bleiben indessen eine Reihe sehr essenzieller kritischer Fragen an die Methode. So fehlt bislang jeder belastbare Nachweis dafür, wie und über wen eine solche Instrumentalisierung im I3. und I4. Jahrhundert gesteuert wurde. Das ändert sich bekanntlich etwa in der Zeit Kaiser Maximilians I., aus der wir ja recht genau wissen, mit welchen Gelehrten und Beratern die Erhaltung des ,Gedächtnis' in verschiedensten Medien umgesetzt wurde. Außerdem ist, wie Matthias Weniger jüngst konstatierte, die Kontextualisierung der meisten von Suckale in Anspruch genommenen Werke, insbesondere ihre Zuweisung an bestimmte politische Akteure, höchst schwierig und oftmals sehr hypothetisch. ${ }^{12}$ Sodann müsste man voraussetzen, dass die Zeitgenossen allesamt ausgebildete kunsthistorische Mediävisten, und zwar kennerschaftlicher Schulung, waren, die all das Fränkische, Oberrheinische, Böhmische sofort erkennen und datieren und somit auf den jeweiligen Herrscher zu beziehen vermochten. Vor allem könnte man auch von zeichen- und kommunikationstheoretischer Seite kritisch ansetzen, denn etwa sekundäre Bedeutungsvereinnahmungen oder Aushandlungsprozesse über Deutungshoheiten sind in der Stilsemantik kaum zu vermitteln. Analoges gilt auch für die zugrunde gelegte historiographische Methode, die in hohem Maße

Luxemburg 1310-1437. Ausst.-Kat. Prag 2006, hg. von Jiř́ FAjT, München - Berlin 2006, S. 357-383, hier S. 357-358.

9 Suckale, Hofkunst (wie Anm. 3), S. 160-169.

10 Dieter KIMPEL - Robert Suckale, Die gotische Architektur in Frankreich. 1140-1270, München 1985.

11 Jiř́ Fajt, Von der Nachahmung zu einem neuen kaiserlichen Stil. Entwicklung und Charakter der herrscherlichen Repräsentation Karls IV. von Luxemburg, in: Karl IV., hg. von Fajt (wie Anm. 8), S. 41-75.

12 Matthias Weniger, Kunst und Hofkunst unter Ludwig dem Bayern, in: Ludwig der Bayer (1314-1347). Reich und Herrschaft im Wandel, hg. von Hubertus SEIBERT, Regensburg 2014, S. 361-384. 
ereignisgeschichtlich verfährt, eben herrscherliche und dynastische Allianzen bzw. Divergenzen politischer und militärischer Natur als wesentliches Paradigma nennt. Die implizit zugrunde gelegten politischen Handlungsmuster bilden insofern Konkurrenz, Gegnerschaft und Absetzung bzw. Loyalität und Parteinahme, während vielfältige Zwischentöne von Kompromiss, Lavieren, Neutralität kaum über Stile vermittelt werden können. Bezeichnenderweise ist bei Suckale bei allem Insistieren auf dem Konflikt zwischen Ludwig und dem Papst bzw. Karl IV. nichts von einer Kompromisspolitik gegenüber dem Habsburger Gegen- oder Mitkönig Friedrich dem Schönen im Felde der Kunst vermerkt. Es sind solche grundsätzlichen Schwierigkeiten, die es neben der lückenhaften dokumentarischen Evidenz als sehr problematisch erscheinen lassen, auf einer Hofkunst Friedrichs zu insistieren und sie in ein Verhältnis zu Ludwig zu setzen.

Derartige Vorbehalte gegenüber einer Verortung stilistischer Idiome von ,Hofkunst' als politisches Herrschaftsinstrument sind bereits von Malcolm Vale oder Wolfgang Brückle formuliert worden. ${ }^{13}$ Wie aber der Verweis auf Kaiser Maximilian deutlich macht, kann den Artefakten sehr wohl eine große Bedeutung bei der vielfältigen Vermittlung politischer und religiöser Selbstverständnisse um 1500 zukommen. Insofern erscheint es sinnvoller, ,Hofkunst ' in einem erweiterten Sinne zu begreifen, nämlich als Artefaktproduktion, die eingebunden ist in ein komplexes System symbolischer Kommunikation, das vor allem auch die Inszenierung von Schauspielen, Turnieren, Einzügen, Banketten, Festen, die literarische und chronikalische Produktion, das Stiftungswesen u. v. m. umfasst. Man kann etwa für die französischen Apanagefürstentümer des I5. Jahrhunderts sehr spezifische Identitätsausbildungen feststellen, die sich künstlerisch vielfältig niederschlagen und bei denen zumindest fallweise auch nachzuweisen ist, dass damit eine zentralisierte und institutionalisierte Verwaltung korreliert war. Wie etwa Simona Slanička gezeigt hat, betrieben die Burgunder seit dem späten I4. Jahrhundert eine intelligente und logistisch kleinteilig geregelte Politik der Embleme und Devisen. In ihrer unmittelbaren Verständlichkeit suggerierten etwa die heraldischen Zeichen von Feuerstahl und Knotenstock militärische Energie und Stärke und erzeugten in ihrer abundanten Omnipräsenz eine unentrinnbare Sphäre von politischer Programmatik, materialisiert etwa in der Ikonographie von Tapisserien, Handschriften oder Neujahrsgeschenken. ${ }^{14}$ Das verbindet sich mit dem programmatischen Insistieren auf dem Troja-Stoff, der literarisch und bildnerisch,

13 Vale, Princely Court (wie Anm. 1), S. 247-252; Brü Ckle, Revision (wie Anm. 1).

14 Simona SlaničKa, Krieg der Zeichen. Die visuelle Politik Johanns ohne Furcht und der armagnakisch-burgundische Bürgerkrieg (Veröffentlichungen des Max-Planck-Instituts für Geschichte 182), Göttingen 2002. 
insbesondere im Rahmen des Ordens vom Goldenen Vlies, evoziert wurde. Dies ließ sich einerseits auf die angemaßte Anziennität der Burgunder beziehen und zeigte, andererseits die Befreiung des Heiligen Landes als politische Programmatik proklamierte und insoweit christlich fundierte Legitimität an. Die Anjou hingegen setzten zur gleichen Zeit deutlich auf eine Entmilitarisierung ihres Images, indem sich der Herrscher selbst als gebildeter Literat präsentierte, Turniere zu literarischen Bukolika transformierte oder herrscherliche Frömmigkeit als eremitengleiche Askese vermittelte. ${ }^{15}$ Dabei spielten literarische und bildnerische Verfahren eine besondere Rolle, die den Teilnehmer/Betrachter/Leser in Art einer Rezeptionsästhetik in die zierlichen Luftschlösser des rex literatus involvierten beim Schäferspiel genauso wie bei der Lektüre von allegorischen Ritterromanen oder Turnieranweisungen.

Als Korrelat derartiger Kreationen politischer Images über symbolische Kommunikationsprozesse können wir in den französischen Fürstentümern dieser Zeit auch die Etablierung von Verwaltungsstrukturen nachvollziehen, die unabhängig von zünftischen Strukturen auf die Person des Fürsten bzw. das von $i$ mm regierte Gemeinwesen orientiert waren. Als sehr gut organisierter Hof zeigt sich gemäß den Recherchen von Thomas Rapin derjenige des Herzogs von Berry. Entscheidend dabei ist die Existenz einer unabhängigen Rechnungskammer, die Chambre de comptes, über die eine stark hierarchisiert strukturierte Bautätigkeit im gesamten Herzogtum bezahlt und kontrolliert wurde. An der Spitze stand als oberster Werkmeister Guy de Dammartin (général maître d'oeuvres du duc), von der Gründung des Herzogtums 1369 bis zu seinem Tod 1397, gefolgt bis I4I3 von seinem jüngeren Bruder Dreux. Diese obersten Werkmeister beaufsichtigten die Baustellen und sicherten zumeist über ein System aus Unter- und Werkverträgen eine zuverlässige Bauführung vor Ort. Der Amtsinhaber führte den Titel Valet de Chambre (der aber vor allem finanztechnischer Natur war!) und verfügte über mehrere Kammerherren, gehörte aber nicht zu den familiares des Herzogs und war, im Unterschied zu Raymond du Temple in Paris und Vincennes, kein Intimus des Herrschers. Guy de Dammartin erhielt eine überdurchschnittliche, aber nicht exorbitante Bezahlung. Im Falle der neu errichteten Herzogsresidenz in Poitiers wissen wir, dass die Tour Maubergeon

15 Christian Freigang, Fantaisie et Ymaginacion: Selbstreflexion von Höfischkeit am provençalischen Hof unter René I., in: Hofkultur in Frankreich und Europa im Spätmittelalter, hg. von DeMs. - Jean-Claude Schmitt (Passagen/Passages 11), Berlin 2005, S. 209-243; DERS., Le tournoi idéal: la création du bon chevalier et la politique courtoise de René d'Anjou, in: René d'Anjou, écrivain et mécène (1409-1480), hg. von Florence BouCHET (Texte, Codex \& Contexte 13), Turnhout 2011, S. 179-196. 
von einem untergebenen, gleichwohl aufgrund seiner Kompetenz privilegierten Werkmeister Jean Guérart auf einem großen Pergament gerissen und durch Guy dem Herzog zur Abnahme vorgelegt wurde, wofür offenbar auch ein Modell aus Nussbaum angefertigt worden war. ${ }^{16}$ Auf den eigenhändigen Entwurf von Guy gehen offenbar auch Hauptwerke wie die Schlösser in Mehun-sur-Yèvre und Poitiers zurück. Wir haben es also mit einer effizient geregelten Bauverwaltung zu tun, innerhalb derer offenbar auch die Entwurfstätigkeit vonstattenging bzw. -gehen konnte. Ein besonderes Augenmerk kam dabei dem Aspekt der Wirtschaftlichkeit zu, der sich etwa darin äußert, dass Kostenvoranschläge, Unterverträge und Baudurchführung über die Chambre de Comptes kontrolliert wurden. Allerdings scheint der Herzog kaum direkt in diese intensive Bautätigkeit involviert gewesen zu sein. Gleichwohl zeichnet sich seine Herrschaft durch eine intensive Bautätigkeit aus: Die Saintes Chapelles in Bourges und in Riom, die Palastum- und -ausbauten in Poitiers, Mehun-sur-Yèvre und des Hôtel de Nesle in Paris sind hier zu nennen. Ein Vergleich der beiden Saintes Chapelles in Riom und in Bourges macht aber klar, dass trotz der analogen Rückbezüge auf das Pariser Vorbild eben kein einheitlicher Stil angewandt wurde, denn zu sehr unterscheidet sich die innovative Formensprache in Riom von derjenigen in Bourges. Die intensive, administrativ effizient geregelte Bautätigkeit im Herzogtum bedeutete offenbar nicht eine stilistische Homogenität.

Diese zentralisierte fürstliche Bauverwaltung erlaubte große Flexibilität, was die persönliche Anteilnahme des Herrschers an der künstlerischen Produktion betrifft. Während - im Gegensatz zur Situation in Paris - zu den Bauleuten keine engeren Bande bestanden, gab es ausgesuchte Maler, die in der unmittelbaren Entourage des Jean de Berry zu verorten sind. Die weidlich bekannten Kronzeugen dafür stellen die Maler und Goldschmiede Paul, Hermann und Hans von Limburg dar - unter denen vor allem Paul eine herausragende Rolle am Hofe spielte -, immer wieder mit Neujahrsgeschenken bedacht und ihrerseits auch als Schenkende auftretend. Wenn sie dabei ein virtuos geschaffenes Salzfass und eine Buchattrappe übergaben, so wiesen sie implizit auf die Gründe ihrer Wertschätzung hin: handwerkliche Qualität und eine überraschende Fähigkeit der bildnerischen Mimesis. Wiewohl die Einzigartigkeit dieser Übergabe von étrennes nicht überbewertet werden darf - denn auch andere Handwerker übergaben an Neujahr Geschenke an den Herrscher -, so fallen doch eine Reihe von direkten herzoglichen Interventionen zugunsten Pauls und seiner Brüder auf: von Ehrentiteln über

16 Thomas Rapin, Les Dampmartin, une dynastie de maîtres d'oeuvre à la lecture des sources (1365-1469), in: Revue historique du Centre-Ouest 4 (2005), S. $247-271$, hier S. 259. 
die Zuführung einer Braut (in Form einer minderjährigen Bürgerlichen) bis hin zur Schenkung eines palastähnlichen Wohn- und Atelierhauses in Bourges. ${ }^{17} \mathrm{Wie}$ schon zuvor am burgundischen Hof waren die Brüder vertraglich auf Arbeiten für den Herrscher festgelegt, und ihr Name wurde zusammen mit demjenigen von Jacquemart des Hesdin insofern zu einer Art Markenzeichen, als die von ihnen ausgestatteten Bücher im Bibliotheksinventar zumindest fallweise explizit als ihre Werke verzeichnet sind. Die Limburgs erscheinen insofern als exemplarisch für einen Hofkünstler, der nun auch in der Tat politische Botschaften vermittelt: Dazu zählt etwa die bildliche Dokumentation der ,Baupolitik' des Herrschers, wie sie in den Veduten des berühmten Stundenbuchs der Très Riches Heures sichtbar wird, die vor allem die fürstlichen Residenzen und Ländereien zeigen und sie in ein höfisch-geziertes Ambiente integrieren. ${ }^{18}$ Die Programmatik der Très Riches Heures muss dabei wohl auf ein differenziertes Konzept, welches sich nicht allein den Künstlerbrüdern verdankt, zurückzuführen sein. Darüber hinaus vermitteln die kosmologischen Darstellungen und Eintragungen in den Kalenderblättern (v. a. $f^{\circ} 2 v, 3 v, 6 v, 7 v, 9 v, 10 v, 1 I v, 12 v$ ), der astronomische Mensch (f $f^{\circ} 14 v$ ) oder die Romkarte ( $f^{\circ}$ I $1 \mathrm{IV}$ ) Gelehrsamkeit als durchgehendes herrscherliches Ideal. Hinzu treten subtile tagespolitische Parteinahmen, wenn etwa die Peiniger Christi in den Passionsdarstellungen ( $f^{\circ}$ I 43r, I4 6v, I 47r, I53r) durch ihre kreuzweisen Schärpen als feindliche Burgunder kenntlich gemacht sind. ${ }^{19}$

Bemerkenswerterweise folgt die Struktur der Bauverwaltung im Berry in vielen Elementen administrativen Neuerungen, die unter König Karl V. am französischen Hof eingeführt worden waren. Die Einrichtung eines général maître des oeuvres du roi als Oberaufsicht der königlichen Baumaßnahmen ist hier ebenso zu nennen wie deren Bezug auf eine eigenständige Rechnungskammer. In diesem System agierte ein mittlerweile gut dokumentierter Kleriker Guillaume d'Ogier als oberster Offizier, dem insbesondere ein Spezialist, Raymond du Temple, als Bauunternehmer und Architekt direkt untergeordnet war. ${ }^{20}$ Dieser, zunächst an

17 Die Brüder van Limburg: Nijmegener Meister am französischen Hof (1400-1416). Ausst.Kat. Nijmegen, hg. von Rob Dü CKERS, Stuttgart 2005, passim.

18 Chantilly, Musée Condé, MS. 65; Les très riches heures du duc de Berry: Manuskript Nr. 65 aus den Sammlungen des Musée Condé in Chantilly, 2 Bde., Luzern 1984; Les très riches heures du Duc de Berry et l'enluminure en France au début du XVe siècle. Ausst.-Kat. Chantilly 2004, hg. von Patricia Stirnemann, Paris 2004.

19 SlaničKa, Krieg (wie Anm. 14), S. 242-280.

20 Jean Chapelot, Charles V maître d'ouvrages: à propos de la construction du donjon de Vincennes et de quelques chantiers contemporains, in: Du projet au chantier. Maîtres d'ouvrages et maîtres d'oeuvre aux XIV ${ }^{\mathrm{c}}-\mathrm{XVI}^{\mathrm{c}}$ siècles, hg. von Odette CHAPELOT (Civilisations et sociétés 106), Paris 2001, S. 337-403, bes. S. 366-368. 
der großen Wendeltreppe des Louvre beschäftigt - an der auch der nachmalige Hauptbauaufseher der Berry, eben Guy de Dammartin, tätig war -, scheint sich in ähnlicher persönlicher Nähe zum König wie die Brüder Limburg bewegt zu haben. Immerhin übernahm dieser höchstpersönlich die Patenschaft eines der Kinder des Baumeisters. Der König selbst richtete zur Förderung bestimmter Projekte, wie etwa dem vielfach veränderten, immer wieder durch persönliche Interventionen geprägten Ausbau des Donjon in Vincennes, einen persönlichen Sonderfonds, die sogenannten coffres, ein und war selbst erstaunlich häufig an Besichtigungen und Begutachtungen von Bauprojekten beteiligt. Dabei handelt es sich insbesondere um Profanarchitekturen, also Fortifikationen, Brücken u. Ä., nicht unbedingt um ästhetisch aufwendige Anlagen. Raymond du Temple spielte hier eine herausragende Rolle, und es war wohl diese exklusive Stellung, die ihm erlaubte, sich am Grand vis du Louvre in einem skulptierten Porträt abbilden zu lassen und sich als familiaris in die dynastische Reihe des Königshauses einzureihen. ${ }^{21}$ Aussagekräftig sind in diesem Zusammenhang auch neuere Erkenntnisse zu seiner Entwurfstätigkeit. Gemäß einer jüngst entdeckten Quelle gab er von Paris aus die Erbauung der Sainte-Chapelle in Vincennes durch Risse und Schablonen vor, die dann von kompetenten Baumeistern ausgeführt wurde. ${ }^{22}$ Damit war sichergestellt, dass das unter Karl begonnene Architekturwerk auch unter seinem Nachfolger gemäß einer zentralisierten Entwurfsplanung ausgeführt werden konnte. Die Quellen für eine derart detailliert geregelte Bauverwaltung lassen sich aber offenbar nicht so lesen, als ob hier ein bestimmter Hofstil als Distinktionsmerkmal bestimmter Gruppen kreiert werden sollte - selbst wenn dies als Sekundäreffekt nicht auszuschließen ist. Die Hauptmotivation lag darin, die herrscherliche Förderung der Produktion von Bildern und Architekturen gezielt herauszustellen.

Wolfgang Brückle hat in diesem Sinne nämlich deutlich gemacht, wie im letzten Drittel des I4. Jahrhunderts unter dem französischen König Karl V. ein neues, profanes Stadt- und Staatsverständnis wirksam wurde. Die zunehmende Ausdifferenzierung der Metiers einerseits, die Orientierung der Politik am bien publique andererseits gab dem Herrscher auch eine bislang nicht zu findende Verantwortung in architektonisch-künstlerischen Fragen. Der umfassende Umbau von Paris zu einer architektonisch prächtigen Kapitale, wie sie unter Karl V. offensichtlich

21 Zuletzt Isabelle Taveau-Launay, Raymond Du Temple, maître d'œuvre des rois de France et des prince, in: Du projet, hg. von Chapelot (wie Anm. 20), S. 323-338; Odette Chapelot - Jean Chapelot - Jean Pascal Fou Cher, Un chantier et son maître d'oeuvre: Raymond Du Temple et la Sainte-Chapelle de Vincennes en 1395-1396, in: Du projet, hg. von Chapelot (wie Anm. 20), S. 433-488.

22 Ebd., bes. S. $459-475$. 
als Politik betrieben wurde, hatte zum Ziel, Schönheit, Pracht und Ordnung des urbanistischen Stadtgefüges als Ausweis einer zunehmend auf Weltliches gerichteten königlichen Politik visuell erfahrbar werden zu lassen. Zu nennen sind hier vor allem die Vergrößerung des Stadtgebietes, der Ausbau des Louvre, des Hôtel Saint-Pol und des Schlosses von Vincennes, zahlreiche Kirchengründungen usw. Seither ist eine gleichsam ,touristische' Inszenierung der schönen Stadt auch Teil von Herrschaftsritualen, wie vor allem der Bericht vom Empfang von Kaiser Karl IV. in Paris deutlich macht. Brückle fasst das als ästhetische Überhöhung des französischen Königtums auf, in dem sich der Staat über die Kunstwerke repräsentiert und damit die Ranghöhe des Herrschers legitimiert. ${ }^{23} \mathrm{Um}$ I 400 wird Christine de Pizan Karl V. unter Rückbezug auf Fürstenideale, die bei Aristoteles und Thomas von Aquin formuliert sind, aufgrund seiner vollendeten Beherrschung der artes liberales als, sage roi' preisen: Charles fust sage artiste, se demoustra vray architecteur et deviseur certain et prudent ordeneur, lorsque les belles fondacions fist faire en maintes places, notables edifices beaulx et nobles, tant d'eglises comme de chasteaulx et autres bastimans à Paris et ailleur $[\ldots] .^{24}$

Versuchen wir nunmehr, die eben genannten Aspekte einer zentralisierten und autonomen ,Bau- und Kunstverwaltung' auf das Reich zu übertragen, so gibt es zunächst eine Reihe von parallel zu lesenden Indizien. So erscheinen meines Wissens zum ersten Mal unter Karl IV. Maler als familiares des Herrschers, nämlich Nikolaus Wurmser aus Straßburg und Meister Theoderich. ${ }^{25}$ Wir können annehmen, dass dazu auch die Dombaumeister Matthias von Arras und Peter Parler gehörten, denn immerhin erscheinen sie innerhalb der von Kaiser Karl IV. angeführten Stiftergemeinschaft, die als Büsten im Prager Domtriforium abgebildet ist. In dieser Funktion sind auch beide bemerkenswerterweise im Dominneren unter ihren Büsten begraben. ${ }^{26}$ Das erinnert an die wenig ältere Aufnahme von Raymond du Temple in die Ehrenplätze am Pariser Grand vis. Peter Parler sind zudem sicher mehrere kaiserliche Bauprojekte zuzuweisen, so außer dem Prager

23 Wolfgang BRÜ CKLE, Civitas terrena. Staatsrepräsentation und politischer Aristotelismus in der französischen Kunst 1270-1380, München - Berlin 2005, bes. S. 166-199.

24 Suzanne Solente, Le livre des fais et bonnes meurs du sage roy Charles V par Christine de Pisan, Bd. 2 (Société de l'Histoire de France, série ant. à 1789, t. 437 et 444), Paris 1936 u. 1940, S. 37.

25 Antonín Fried L, Magister Theodoricus. Das Problem seiner malerischen Form, Prag 1956, S. $24-25$ u. S. 333-334.

26 Christian Freigang, Werkmeister als Stifter. Bemerkungen zur Tradition der Prager Baumeisterbüsten, in: Nobilis arte manus. Festschrift zum 70. Geburtstag von Antje Middeldorf Kosegarten, hg. von Bruno KLein - Harald Wolter-von DeM KNeSEBECK, Dresden Kassel 2002, S. 244-264. 
Dom auch der Altstädter Brückenturm und die Allerheiligenkirche auf dem Hradschin. Derartig hochrangige Aufträge machten Parler wie auch seine französischen Kollegen zu wohlhabenden Grundbesitzern. Angesichts solcher generellen Vergleichbarkeiten fällt aber umso mehr auf, was wir im Gegensatz zu den französischen Quellen dokumentarisch entbehren: keine Titel innerhalb einer höfischen Verwaltung, keinerlei Angaben zu zentralisierenden und institutionalisierenden Strukturen oder Finanzierungsmodi. Im Gegenteil sind beide Meister des Prager Doms in ihren Inschriften ausdrücklich nur als Magister fabricae des Doms genannt. ${ }^{27}$ Andererseits ist sicherlich erwägenswert, dass die umfangreiche, durch Karl in Angriff genommene Erweiterung der Prager Altstadt durch die Anlage der Neustadt mit dem zentralen Wenzelsplatz eine umfangreiche Logistik und zentrale Finanzierung voraussetzte, in die wohl auch hoch qualifizierte Werkmeister involviert waren, die dem Herrscher nahestanden und ihre Kompetenzen in entscheidendem Maße einfließen ließen. Hierfür wurde jüngst von einigen Autoren konkret Matthias von Arras in Anspruch genommen, ohne dass dies indessen wie zur selben Zeit in den französischen Fürstentümern im Sinne einer Kunst- und Bauverwaltung quellenmäßig greifbar wäre. ${ }^{28}$ Analoges gilt für die ausgeklügelte Inszenierung des Prager Doms als Ritual- und Memorienort Karls IV., die nicht ohne eine koordinierende Planung zu denken ist. ${ }^{29}$

Vergleichbares können wir für Ludwig IV., den Bayern, nicht ermitteln. Im Gegenteil ist die Anzahl seiner Stiftungen und der von ihm in Auftrag gegebenen Bauten und Kunstwerke eher gering: die Lorenzkapelle im Alten Hof in München, der Chor und der Hauptaltar sowie die wittelsbachische Grablege in der Münchener Frauenkirche, das Kloster Ettal, die Ettaler Madonna, die Fürstenfelder Madonna. ${ }^{30}$ Bis auf die beiden erhaltenen - stilistisch völlig unterschiedlichen

27 Zur Problematik der Inschriften im Chortriforium: Milena BART LovÁ, The Choir Triforium of Prague Cathedral Revisited: The Inscriptions and Beyond, in: Prague and Bohemia. Medieval Art, Architecture and Cultural Exchange in Central Europe, hg. von Zoë Opačıć (Conference Transactions. The British Archaeological Association 32), Leeds 2009, S. 81 - 100; zur Bauinschrift am Turm: Klára BenešovsKÁ - Ivo HLOBIL, Peter Parler \& St Vitus's Cathedral 1365-1399, Prag 1999, S. 149-151.

28 Zoë OpaČić - Paul Crossley, Prague as a New Capital, in: Prague. The Crown of Bohemia, 1347 - 1437, hg. von Barbara Drake Bоенм - Jiří FAjt, New York u. a. 2005, S. 59-73, hier S. 66-67.

29 Paul Crossley, Bohemia sacra: Liturgy and History in Prague cathedral, in: Pierre, lumière, couleur. Etudes d'histoire de l'art du Moyen Âge en l'honneur d'Anne Prache, hg. von Fabienne Joubert - Dany Sandron, Paris 1999, S. 341-365.

30 Weniger, Kunst (wie Anm. 12); eine Übersicht bei Peter Pfister, Staatsfrömmigkeit und Privatfrömmigkeit Ludwigs des Bayern in seinem bayerischen Herrschaftsgebiet, in: 
Kunstkreisen entstammenden - Madonnen sind die den Stiftungen zugehörigen Ensembles verloren bzw. stark beeinträchtigt. Angaben über die beteiligten Künstler, ihre Verbindung mit der kaiserlichen Verwaltung fehlen ganz. Etwas besser stellt sich die Situation für die Dynastie der konkurrierenden Luxemburger dar, der ja auch Ludwigs Vorgänger, Heinrich VII., entstammte. Vor allem der Trierer Erzbischof Balduin von Luxemburg, Bruder Heinrichs VII. und Großonkel Kaiser Karls IV., ist gut erforscht. ${ }^{31}$ Die Vielfalt und der Umfang der Stiftungen sicherten dem Geschlecht ein intensives und lang währendes Andenken. Auffällig ist bei Heinrich VII. eine geographisch äußerst weite Streuung der Stiftungen und Memorialorte zwischen Trier, dem Mittelrhein und Mittelitalien. Dies war natürlich nicht zentral zu organisieren, sondern wurde von lokalen Kräften, dem Pisaner Domkapitel oder dem Oberweseler Stiftskollegium, eben den von den Stiftungen profitierenden Institutionen, betrieben. Dabei war die künstlerische Ausgestaltung im Grunde eine nachgeordnete Frage, selbst wenn natürlich solch hochrangige Aufträge von exquisiten Ateliers auszuführen waren. Entscheidende Bedeutung kam den vermittelten Images zu - wie das Michael Viktor Schwarz genannt hat: ${ }^{32}$ die Referenz auf antikes Kaisertum in Pisa etwa oder die Legitimität des Handelns in der Romfahrt Heinrichs. Daraus rechtfertigte sich die Memoria durch bestimmte, bewusst diversifizierte Personengruppen. Analoges gilt für die Vielfalt der Stiftungen von Balduin von Trier, die sich indessen wegen der Komplexität der damit verbundenen Motive nicht unter dem Rubrum einer morphologisch einheitlichen Hofkunst einordnen lassen. ${ }^{33}$ Vielmehr scheint es auch hier, als hätten die bestifteten Institutionen die bildlich- oder baulichmaterielle Umsetzung selbst in die Hand genommen.

Erst der neue Akzent auf die Herausstellung einer guten künstlerischen Qualität als Ausdruck eines geordneten bien publique, die unter Karl V. von Frankreich betrieben wurde - eine Politik, in die der Herrscher explizit auch direkt involviert

Kaiser Ludwig der Bayer 1282 - 1347. Ausst.-Kat. Fürstenfeldbruck 1997, hg. von Angelika Mundorff - Renate WedL-BRu ognolo, Fürstenfeldbruck 1997, S. 53-76.

31 Franz J. Ronig, Kunst unter Balduin von Luxemburg, in: Balduin von Luxemburg, Erzbischof von Trier - Kurfürst des Reiches. 1285-1354, hg. von Johannes Mö s CH - Franz-Josef HEYEN (Quellen und Abhandlungen zur mittelrheinischen Kirchengeschichte 53), Mainz 1985, S. 489-558; Grabmäler der Luxemburger. Image und Memoria eines Kaiserhauses, hg. von Michael Victor Schwarz, Luxemburg 1997; Verena Kessel, Balduin von Trier (1285-1354). Kunst, Herrschaft und Spiritualität im Mittelalter (Geschichte und Kultur des Trierer Landes 12), Trier 2012.

32 Michael Victor Schwarz, Image und Memoria: Statt einer Zusammenfassung, in: Schwarz, Grabmäler (wie Anm. 31), S. 175-182.

33 KesseL, Balduin (wie Anm. 31), bes. S. 399-400. 
erscheint -, begründete offenbar eine neue zentralisierende Koordination des künstlerischen und architektonischen Schaffens. Ob damit ein einheitlicher künstlerischer Stil kreiert wurde, bleibt weiterhin eine Frage. Jedenfalls machte diese neue Koordination recht schnell Schule, in den französischen Apanagefürstentümern und in Ansätzen auch im Reich, und muss als Innovation dieser Zeit gelten, von der die Frage einer konzertierten ,Hofkunst' in der ersten Hälfte des I4. Jahrhunderts abgesetzt werden sollte. 